\title{
Sintomatologia em mães de adolescentes: Comparação entre praticantes e não praticantes de actividade física
}

\section{Symptomology among mothers of adolescents: Comparison between physically active and non-active mothers}

\begin{abstract}
RESUMO
O principal objectivo deste estudo foi verificar se a prática de exercício físico poderia influenciar os sintomas breves em mães de adolescentes nas diferentes fases de desenvolvimento. Foi usada uma amostra de 180 mulheres, com idades compreendidas entre os 31 e os 55 anos (42.32 \pm 5.49$)$, tendo pelo menos um filho adolescente. O instrumento utilizado foi o BSI18 (Brief Symptom Inventory - 18) de Canavarro (1999). Os resultados sugerem, como está referido na literatura, que os indivíduos que praticam actividade física apresentam menores níveis de ansiedade, depressão, somatização e índice geral de sintomas (IGS) do que indivíduos que não sigam tais recomendações. Nas variáveis: número de filhos, estado civil e fases da adolescência não se denotaram diferenças estatisticamente significativas, porém mães divorciadas e com maior número de filhos apresentam piores índices de saúde mental. As que praticam exercício físico têm melhor saúde.
\end{abstract}

Palavras-chave: sintomas breves, actividade física, adolescência, maternidade

\section{ABSTRACT}

This study's main objective is to explain how physical exercise can influence the brief symptoms in mothers of adolescents in different stages of development. The sample consisted of 180 women with at least one of her children passing through this phase, aged between 31 and $55(\mathrm{X}=42.32)$. The instrument used to evaluate the variables was the BSI18 (Brief Symptom Inventory - 18) of Canavarro (1999). The results suggest, as refer during the literature, the people who practice physical activity had lower anxiety, depression, and somatization levels than those who do not achieve such recommendations. In the variables: "son's number", "civil state" and "adolescence states". However, divorced mothers with more children have higher rates of mental health. Those who practice physical exercise have better health.

Keywords: symptoms brief, physical activity, adolescence, maternity 
Ao longo dos tempos a mulher tem vindo a assumir um papel mais activo na sociedade, isto fez com que tivesse a responsabilidade de um emprego e do cuidar dos filhos. Assim as mulheres tiveram que demonstrar que desempenhavam diferentes tarefas da mesma maneira que os homens, isto é, a mulher ganhou uma certa autonomia perante os diferentes papéis impostos pela sociedade.

Contudo educar um filho com 4 anos de idade não será terá o mesmo grau de exigência de quando se trata acompanhar o crescimento de um jovem adolescente. Este é um período do desenvolvimento em que os indivíduos sofrem alterações tanto a nível psicológico como físico. Por isso será pertinente verificar que sintomas podem desenvolver as mães tendo a seu cargo filhos adolescentes, pois é nesta altura que o jovem pretende adquirir autonomia, afastando-se, de alguma forma, do meio familiar, querendo explorar o meio psicosociocultural que o rodeia. Esta posição do adolescente face à mãe pode, por vezes, não ser bem entendida e por essa razão ser geradora de conflitos entre eles. Estes conflitos, muitas vezes, podem trazer consequências a nível psicológico para as mães, podendo-se traduzir em depressões, perturbações de ansiedade e somatização. Tendo isto em conta, é importante verificar se a variável actividade física (prática ou não) tem alguma repercussão no desenvolvimento destes sintomas, e como o número de filhos pode acentuar a prevalência desses sintomas.

Hoje em dia o termo "adolescente" é usado espontaneamente, no entanto nem sempre foi assim, só no século XX se deu importância a esta nova fase do desenvolvimento tanto a nível científico como no senso comum. A adolescência pode ser definida como "uma fase de reestruturação afectiva e intelectual da personalidade, um processo de individualização e de metabolização das transformações fisiológicas ligadas à integração do corpo sexuado" (Doron \& Parot, 2001, p.32).
Segundo o Relatório da organização mundial da saúde a adolescência está dividida em três fases, que se inicia aos 11 anos e termina nos 21 anos, sendo a primeira fase denominada por fase inicial que se situa dos 11 anos e os 14 anos. É nesta fase que o jovem procura estabelecer relações entre pares afastando-se de uma certa maneira da sua família; a segunda fase, chamada de fase intermédia começa nos 14 anos e vai até aos 17. è durante esta fase que tende a acorrer uma intensa reorganização psicológica havendo, no entanto, alguma persistência dos estados de humor e comportamentos desajustados; finalmente, a última fase designada por fase final, enquadra os 17 anos estende-se até aos 20 anos. É nesta altura que o jovem manifesta um reconhecimento do seu papel enquanto ser social (Borsa \& Grendene, 2008).

A adolescência implica importantes mudanças em várias dimensões psicológicas estando estando estas associadas a grandes riscos. Alguns jovens têm dificuldades em lidar com tantas mudanças em tão pouco tempo e perante essa realidade podem precisar de ajuda, para ultrapassar os conflitos que lhes surgem no seu dia-a-dia. É no processo de ultrapaqssar estas dificuldades que os pais podem ter um papel fundamental, auxiliando-os a compreender as falhas próprias de quem ainda não atingiu a maturidade nas suas escolhas (Feldman, Olds, \& Papalia, 2001).

A estrutura familiar é essencial, pois esta constitui uma ligação entre o indivíduo e o mundo que o rodeia, não deixando de lado a importância da sua individualidade e singularidade. Deste modo, a família está subjacente a um desenvolvimento favorável tanto cognitiva como emocionalmente (Brito, 2006). É de salientar que a função da família é enquadrar o adolescente melhorando, desta maneira, a sua qualidade de vida (Jatobá \& Bastos, 2007).

A família como instituição tem vindo a modificar-se, sendo importante não se dissociar da sociedade, uma vez que dependem 
uma da outra, denotando-se uma complementaridade entre ambas. Nunca esquecendo que a sociedade em que o individuo está inserido compreende várias dimensões humanas, sendo elas, "biológica, psicológica, social, cultural, económica, legal, política e religiosa" (Brito, 2006, p.27).

É fundamental detetar quais os fatores perturbadores que têm vindo a afectar desfavoravelmente o desenvolvimento do adolescente. Inevitavelmente este estudo será uma reflexão sobre os sintomas da mãe que traz consigo inúmeras complicações, tanto a nível emocional, comportamental e cognitivo (Mendes, Loureiro, \& Bastos, 2008).

Segundo o DSM V (2014), a somatização consiste em sintomas físicos de origem psicológica, ou seja, é uma perturbação do foro mental expressando-se de forma física. Relativamente à depressão, é de referir que esta tem sido caracteriza pela fusão de um humor depressivo e de uma diminuição psicomotora podendo até mesmo conduzir à ideação suicida (Doron \& Parot, 2001). Sendo de grande relevância considerar que este transtorno psiquiátrico tem predominância na população feminina, e tem-se verificado que a prevalência aumenta com a idade. Assim, prevê-se que a depressão atingirá, pelo menos uma vez na vida entre 10 a $20 \%$ das mulheres. Daí surge a importância de estudar a depressão como referência para um provável transtorno psicossocial na vida da criança e, posteriormente, a sua adolescência e vida adulta, podendo até ser visto como uma condição de fragilidade (Mendes, et al., 2008).

De acordo com Vasconcelos-Raposo, Fernandes, Mano e Martins (2009), a depressão e o exercício físico são duas variáveis que apresentam uma relação inversa. Quando uma das variáveis aumenta, a outra tende a diminuir significativamente. De acordo com os resultados obtidos, estes pesquisadores verificaram que a depressão é, em média, superior nos indivíduos que não atingem os níveis de exercício físico recomendado quando comparados com os que atingem os níveis recomendados. Também foi constatado que os valores médios de depressão diminuem à medida que se passa para uma categoria do exercício físico no qual o indivíduo é mais activo, podendo-se concluir que o exercício físico contribui, significativamente, para a redução ou prevenção da depressão ou sintomas depressivos.

Os resultados deste estudo demonstram que à medida que se aumenta a prática de exercício físico, os sintomas depressivos tendem a diminuir (Vasconcelos-Raposo, et al., 2009). Para estes autores, a prática desportiva poderá ter um carácter positivo na prevenção ou redução da depressão nos adultos. Outro estudo (Gonzalez, Romero, López, Ramirez, \& Stefanelli, 2010) permitiu uma compreensão do significado e da vivência dos cuidadores destes pacientes que sofrem de depressão e de quanto essas repercussões têm de negativo para a saúde mental do cuidador e do familiar com depressão. Deste modo os cuidadores culpam-se por não terem percebido quais os factores que levaram os familiares ao seu cuidado a desenvolverem sintomas de depressão, sendo este, também um elemento que pode contribuir .para acrescer o risco de eles próprios desenvolverem uma depressão. Por isso o cuidador tem de ter conhecimento sobre o problema em questão, isto é, estar preparado para intervir no processo terapêutico que visa a diminuição das dificuldades enfrentadas aquando da implementação do programa que leva à redução da ansiedade (Gonzalez, et al, 2010).

Outro factor a ser tido em consideração é a ansiedade. O termo ansiedade tem sido caracterizado como impreciso, uma vez que é utilizado em diferentes sistemas explicativos, sob controlo de diferentes eventos (Coelho \& Tourinho, 2008). Esta perturbação é caracterizada por uma inquietação global e um sentimento de tensão sem um motivo específico. $\mathrm{O}$ principal traço que a define, é um medo não realista e excessivo em relação a aconteci- 
mentos futuros. Pode ainda adoptar aspectos somáticos, como dores de estômago e cefaleias (Freitas, 2009).

Relativamente à ansiedade, segundo Araújo, Leite e Mello (2006) expõem evidências da limitada condição de aptidão física dos pacientes com este transtorno, aumentando a predisposição destes indivíduos ao desenvolvimento de outras doenças crónicas e degenerativas além do transtorno de ansiedade. Os autores não apresentam evidências esclarecedoras de que o exercício físico melhora o tratamento dos transtornos de ansiedade, embora sejam mais claros em relação ao transtorno do pânico (Araújo et al. 2006). Já Cheik et al. (2003) confirmam que o programa de exercício implementado demonstrou uma tendência para a redução dos níveis indicativos da ansiedade e da depressão, não tendo, no entanto, alcançado um nível satisfatório que proporcionasse a alteração no grau de severidade da patologia.

Especificamente, em relação aos níveis de depressão, o programa de exercício não foi capaz de atingir um estádio de ausência de níveis criticos para a depressão, ou seja, os indivíduos praticantes de actividades físicas ainda apresentaram indicadores de depressão moderada. Dessa forma, o efeito psicológico e a interacção social proporcionada por este tipo de actividade são válidos, mas se houvesse um ligeir acréscimo na relação volume e intensidade da actividade física realizada, seria possível promover alterações metabólicas e fisiológicas nos indivíduos, e assim, obter um resultado mais satisfatório (Cheik et al., 2003).

No entanto, no grupo dos praticantes de desporto (inclui todas as actividades físicas) verificaram-se resultados mais satisfatórios, no qual foi possível observar que os indivíduos praticantes de exercícios físicos regulares (com frequência, duração, volume e intensidade previamente estabelecidos) diminuíram ainda mais os níveis indicativos para ansiedade e passaram da classificação de levemente deprimidos a não deprimidos. O que foi observado poderia ser atribuído às melhoras fisiológicas e metabólicas decorrentes do exercício físico, como, por exemplo, maior libertação de alguns neurotransmissores como a noradrenalina e a serotonina.

Vários são os estudos que nos dão conta dos benefícios, tanto psicológicos como biológicos da actividade física, ou seja, melhoria da capacidade cardio-respiratória, diminuição de sintomas vasomotores, da pressão arterial, da incidência de doenças cardiovasculares, prevenção do aparecimento de diabetes, da osteoporose, da obesidade, da hipertensão, redução da ocorrência de certos tipos de cancro, aumento de expectativa de vida, entre outros. No aspecto psicológico verifica-se melhoria da auto-estima, auto-conceito, auto-imagem e diminuição da ansiedade e depressão (Salve, 2007).

Estes valores mostraram variar a nível do estado civil, observando-se que as mulheres divorciadas manifestam elevados níveis de depressão comparativamente às solteiras. É de referir que mulheres casadas com filhos revelam predominância destes sintomas (Teixeira, 1998).

Tendo por base a revisão da literatura realizada, formularam-se os seguintes objetivos especificos: Comparar por: 1- estado civil; 2por número de filhos; 3- fase da adolescência; 4- prática e não-prática de atividade física das mães ao nível do índice geral de sintomas e respectivos factores (depressão, ansiedade e somatização).

\section{MÉTODO}

O presente estudo é de natureza transversal, visto que os dados foram recolhidos num único momento; e de carácter quantitativo uma vez que se apoia em dados de natureza numérica. Trata-se de um estudo do tipo descritivo, dado que assenta na descrição e exploração dos dados obtidos. É um estudo não-experimental, uma vez que não foram manipuladas variáveis 
independentes e que adoptou uma perspectiva comparativa. As variáveis independentes em estudo são: exercício físico, número de filhos, estado civil e idade, quanto às variáveis dependentes são: somatização, ansiedade, depressão e Índice geral de sintomas.

\section{Amostra}

Para a realização desta investigação contou-se com a colaboração de 180 mulheres (n $=180$ ), com idades compreendidas entre os 31 e 55 anos, residentes no norte interior de Portugal, maioritariamente casadas $(81,7 \%)$ e mães com filhos adolescentes com idades compreendidas entre os 12 e os 21 .

De referir que se trata de uma amostra de conveniência, uma vez que as respectivas participantes foram recrutados na região norte do país, pelos elementos do grupo de investigação, e que apenas foram recrutadas participantes que tivessem filhos adolescentes.

\section{Instrumentos}

A recolha de dados foi estruturada por três componentes/ partes: o questionário sócio-demográfico, o questionário de exercício físico (Prochaska, Sallis, \& Long, 2001) e sua regularidade e o questionário de sintomatologia breve (BSI-18, Canavarro, 1999).

O questionário sociodemográfico inclui as variáveis idade, local de residência (rural/ urbano), estado civil (solteira, casada, em união de facto, divorciada/separada) e número de filhos.

A prática de exercício físico por semana foi inquirida através de 8 opções $(0,1,2,3,4,5$, $6,7)$ correspondendo estas ao número de dias da semana em que a pessoa pratica exercício físico. O referido questionário está validado para a população portuguesa.

Por fim, o questionário usado para avaliar a sintomatologia breve foi a versão portuguesa do BSI-18 (Brief Symptom Inventory - 18) traduzida e adaptada, para a população portuguesa por Canavarro (1999). Neste questionário o indi- víduo avalia a intensidade com que cada um dos dezoito sintomas (correspondentes aos dezoito itens) o afectou nos últimos 7 dias, incluindo o próprio dia de resposta ao questionário, usando uma escala de 5 pontos numa escala de Likert variando de 0 (nunca) a 4 (Muitíssimas vezes) (Chen, et al., 2010).

\section{Procedimentos}

Para a recolha dos nossos dados foi necessário pedir uma autorização do estabelecimento escolar para a aplicação dos questionários, assim sendo, foram contactados o director da Escola e os pais dos discentes abrangidos no estudo. De seguida contactou-se directamente com a professora dos alunos, escolhidos aleatoriamente, de forma a explicar a finalidade do projecto e, por conseguinte, a docente teve de entregar aos alunos de forma a que estes os levassem até às mães, para serem preenchidos e devolvidos em envelope fechado sem qualquer tipo de dados que pudesse levar a identificação de quem havia respondido aos questionários. Todos os envelopes foram entregues aos professores que, posteriormente, os entregaram aos pesquisadores.

Relativamente aos restantes questionários, momentos antes de entregar o questionário com as devidas instruções, explicou-se, de forma clara e imparcial, os objectivos do estudo bem como as possíveis dúvidas que se poderiam colocar. Foram-lhes dadas garantias de anonimato e confidencialidade.

Após as participantes concordarem em colaborar, e a fim de evitar conclusões incorrectas, a resposta aos questionários ocorreu de forma autónoma e anónima, ficando as participantes com os questionários a fim de estes serem preenchidos sem pressões e com todas as condições de privacidade. A maioria das participantes não mostrou qualquer relutância em colaborar com o estudo e de seguida fez-se a respectiva recolha.

Para a análise estatística dos dados foi utilizado a versão 24.0 do SPSS (Statistical Package for Social Sciences). Relativamente à consis- 
tência interna, as quatro variáveis apresentaram valores favoráveis, demonstrando assim a fiabilidade das escalas. Para a depressão registou-se um alpha de Cronbach de .84, para a ansiedade o valor registado foi .79 , somatizaçao apresenta um valor de .74 e o índice geral de sintomas .89 (Chen et al., 2010).

Para a análise descritiva das variáveis e da amostra foi utilizada uma estatística descritiva de tendência central (média, desvio padrão, frequência absoluta e percentagens). Os testes estatísticos utilizados foram: Mann Whitney e Kruskal-Wallis.

\section{RESULTADOS}

Segue-se a apresentação dos resultados obtidos, consoante a ordem das as variáveis apresentadas nos objetivos específicos.

A partir da análise do quadro 1 verifica-se que o nível de depressão em mulheres divorciadas $(5.63 \pm 3.32)$ apresenta valores superiores aos restantes estados civis seguindo-se mulheres casadas, união de facto e as solteiras; relativamente à somatização também não foi valor estatisticamente significativo. No entanto, as mães solteiras apresentam valores ligeiramente superiores.

No que diz respeito à ansiedade, os valores

Quadro 1

Comparação entre o índice geral de sintomas (IGS), somatização, ansiedade e depressão e estado civil (kruskal-Wallis)

\begin{tabular}{lcccccc}
\hline & $\begin{array}{c}\text { Solteira } \\
(n=11) \\
\mathrm{M} \pm \mathrm{DP}\end{array}$ & $\begin{array}{c}\text { Casada } \\
(n=147) \\
\mathrm{M} \pm \mathrm{DP}\end{array}$ & $\begin{array}{c}\text { União de facto } \\
(n=6) \\
\mathrm{M} \pm \mathrm{DP}\end{array}$ & $\begin{array}{c}\text { Divorciada } \\
(n=16) \\
\mathrm{M} \pm \mathrm{DP}\end{array}$ & $Q^{2}$ & $p$ \\
\hline Somatização & $3.09 \pm 2.51$ & $2.39 \pm 2.39$ & $2.33 \pm 1.197$ & $3.00 \pm 2.83$ & 2.01 & .57 \\
Ansiedade & $4.36 \pm 2.25$ & $4.42 \pm 3.14$ & $6.00 \pm 2.97$ & $6.44 \pm 3.61$ & 7.42 & .06 \\
Depressão & $3.36 \pm 2.42$ & $3.93 \pm 3.33$ & $3.83 \pm 3.31$ & $5.63 \pm 3.32$ & 4.69 & .20 \\
IGS & $10.82 \pm 6.06$ & $10.73 \pm 7.62$ & $12.17 \pm 6.27$ & $15.06 \pm 7.30$ & 6.76 & .08 \\
\hline
\end{tabular}

encontrados demonstraram que as mães divorciadas apresentam níveis mais elevados, não sendo estatisticamente significativo mas estando no limiar de significância $\left[\chi^{2}(3)=\right.$
7.42, $p=.06]$, também acontece com o valor que existe uma tendência para que o nível de índice geral de sintomas se manifesta mais em mulheres divorciadas $\left[\chi^{2}(3)=6.76, p=.08\right]$.

Quadro 2

Comparação entre o índice geral de sintomas (IGS), somatização, ansiedade e depressão e o número de filhos (kruskal-Wallis)

\begin{tabular}{lcccccc}
\hline & $\begin{array}{c}\text { 1 Filho } \\
(n=77)\end{array}$ & $\begin{array}{c}2 \text { Filhos } \\
(n=80) \\
\mathrm{M} \pm \mathrm{DP}\end{array}$ & $\begin{array}{c}3 \text { Filhos } \\
(n=17) \\
\mathrm{M} \pm \mathrm{DP}\end{array}$ & $\begin{array}{c}4 \text { Filhos } \\
(n=6)\end{array}$ & $\begin{array}{c}\mathrm{M} \pm \mathrm{DP} \\
Q^{2}\end{array}$ & $p$ \\
\hline Somatização & $2.21 \pm 2.11$ & $2.74 \pm 2.78$ & $2.47 \pm 1.84$ & $2.83 \pm 2.40$ & 1.83 & .61 \\
Depressão & $4.03 \pm 3.06$ & $3.96 \pm 3.52$ & $4.00 \pm 3.35$ & $5.50 \pm 3.33$ & 2.05 & .56 \\
Ansiedade & $4.62 \pm 3.01$ & $4.73 \pm 3.44$ & $4.53 \pm 2.76$ & $4.33 \pm 3.61$ & .04 & .10 \\
IGS & $10.86 \pm 6.50$ & $11.43 \pm 8.58$ & $11.00 \pm 6.79$ & $12.67 \pm 7.94$ & .46 & .93 \\
\hline
\end{tabular}


A partir da análise do quadro 2 relativamente aos níveis de somatização, ansiedade, depressão e índice geral de sintomas não se verificam valores significativos. No entanto, existe uma tendência para as mães com quatro filhos apresentarem valores ligeiramente superiores relativamente ao índice geral $(12.67 \pm 7.94)$.

A partir do quadro 3, relativamente aos níveis de somatização, ansiedade, depressão e índice geral de sintomas não se verificam valores significativos. Embora haja uma tendência para as mães com filhos que se encontram nas três fases da adolescência

Quadro 3

Comparação entre o índice geral de sintomas (IGS), somatização, ansiedade e depressão e o número de filhos (kruskal-Wallis)

\begin{tabular}{|c|c|c|c|c|c|c|c|c|}
\hline & $\begin{array}{l}\text { Fase inicial } \\
\quad(n=74) \\
\mathrm{M} \pm \mathrm{DP}\end{array}$ & $\begin{array}{c}\text { Fase inter- } \\
\text { média } \\
(n=33) \\
\mathrm{M} \pm \mathrm{DP}\end{array}$ & $\begin{array}{c}\text { Fase final } \\
(n=25) \\
\mathrm{M} \pm \mathrm{DP}\end{array}$ & $\begin{array}{c}\text { f.i. +F.int. } \\
(n=25) \\
\mathrm{M} \pm \mathrm{DP}\end{array}$ & $\begin{array}{c}\text { f.i. + F.final } \\
\begin{array}{c}(n=25) \\
\mathrm{M} \pm \mathrm{DP}\end{array}\end{array}$ & $\begin{array}{c}\text { F.i. +F.final } \\
(n=13) \\
\mathrm{M} \pm \mathrm{DP}\end{array}$ & $\chi^{2}$ & $p$ \\
\hline Somat & $2.36 \pm 1.92$ & $2.52 \pm 1.86$ & $2.56 \pm 2.87$ & 2.20 & 3.77 & $2.00 \pm 4.47$ & 5.28 & .51 \\
\hline Depressão & $4.30 \pm 3.34$ & $3.52 \pm 3.49$ & $3.64 \pm 2.83$ & $4.20 \pm 2.66$ & $4.62 \pm 4.87$ & $3.29 \pm 1.98$ & 3.36 & .76 \\
\hline Ansiedade & $4.62 \pm 3.03$ & $4.48 \pm 2.74$ & $4.80 \pm 3.11$ & $4.48 \pm 2.22$ & $5.85 \pm 5.97$ & $2.86 \pm 2.73$ & 3.80 & .70 \\
\hline IGS & $11.28 \pm 6.89$ & $10.52 \pm 6.81$ & $11.00 \pm 6.94$ & $10.88 \pm 5.52$ & $14.23 \pm 14.86$ & $8.14 \pm 6.82$ & 3.40 & .76 \\
\hline
\end{tabular}

apresentarem valores ligeiramente superiores na depressão $(5.00 \pm 4.36)$, e ansiedade $(6.33 \pm 3.51)$. Relativamente à somatização $(3.77 \pm 4.46)$, e índice geral de sintomas $(14.23 \pm 14.86)$, verifica-se uma tendência para as mães que tenham filhos na fase inicial e final da adolescência demonstrarem valores mais altos.

A partir da análise do quadro 4, verifica-se que as mães que praticam actividade física apresentam valores médios inferiores relati- vamente às mães que não praticam, por isso existem diferenças nos níveis de depressão entre mulheres que praticam $(3.18 \pm 2.90) \mathrm{e}$ as que não praticam $(5.22 \pm 3.45)$, de forma estatisticamente significativa, $U_{(104,76)}=180$, $p=.001$; existem diferenças nos níveis de ansiedade entre mulheres que praticam (3.73 $\pm 2.97)$ e as que não praticam $(5.91 \pm 3.04)$, de forma estatisticamente significativa, $U_{(104}$, ${ }_{76)}=180, p=.001$; verifica-se que existem diferenças nos níveis de somatização entre

Quadro 4

Comparação entre o índice geral de sintomas (IGS), somatização, ansiedade e depressão que praticam ou não actividade física. (Mann Whitney)

\begin{tabular}{lcccc}
\hline & $\begin{array}{c}\text { Praticante } \\
(n=104) \\
\mathrm{M} \pm \mathrm{DP}\end{array}$ & $\begin{array}{c}\text { Não praticante } \\
(n=76) \\
\mathrm{M} \pm \mathrm{DP}\end{array}$ & $Z$ & $p$ \\
\hline Somatização & $1.93 \pm 2.08$ & $3.25 \pm 2.64$ & -3.10 & \\
Ansiedade & $3.73 \pm 2.97$ & $5.91 \pm 3.04$ & -5.19 & \\
Depressão & $3.18 \pm 2.90$ & $5.22 \pm 3.45$ & -4.34 & .001 \\
IGS & $8.85 \pm 6.71$ & $14.38 \pm 7.42$ & -5.90 & \\
\hline
\end{tabular}


mulheres que praticam $(1.93 \pm 2.08)$ e as que não praticam $(3.35 \pm 2.64)$, de forma estatisticamente significativa, $U_{(104,76)}=180, p=$ .001; finalmente, na observação de resultados da comparação do índice geral existem diferenças significativas entre mães que praticam $(8.85 \pm 6.71)$ e as que não praticam $(14.38 \pm$ $7.42)$ atividade física, $U_{(104,76)}=180, p=.001$.

\section{DISCUSSÃO}

Globalmente, os resultados do presente estudo foram de encontro aos resultados apresentados pelos diversos autores e respectivos estudos mencionados ao longo da revisão da literatura apresentada na introdução.

As comparações por estado civil, apesar de não produzirem diferenças estatisticamente significativas, com base nos valores das médias obtidas, deixam transparecer que são as mães divorciadas que evidenciam os piores índices de saúde. Estes resultados permitem sugerir que a sobrecarga de trabalho e afetiva se fazem repercutir na saúde mental das mesmas. Seguindo a mesma lógica de argumentação e dando-lhe consistência, verificamos que quantos mais filhos piores os indicadores de saúde.

No que diz respeito ao número de filhos, constatou-se que mães com quatro filhos evidenciavam níveis superiores do índice geral de sintomas. Por falta de literatura que comprove ou refute este resultado, não nos é permitido fazer uma análise de comparação entre as variáveis em questão.

Ao examinar a comparação entre mães que praticam exercício físico ou não, verificou-se que as mães praticantes apresentam valores inferiores para os factores ansiedade, somatização, depressão e índice geral de sintomas a um nível de significância de $p<.001$. Estes resultados corroboram os já anteriormente encontrados por Vasconcelos-Raposo et al. (2009) pois os resultados deste estudo demonstram que à medida que se aumenta a prática de exercício físico, os sintomas depres- sivos tendem a diminuir. Quanto aos níveis de ansiedade estes também tendem a diminuir com a prática de actividade física, como referido por Cheik et al. (2003).

É de salientar que vários são os estudos que referem os benefícios da actividade física tanto ao nível psicológico como fisicamente. Salve (2007) menciona que para além dos benefícios físicos, os aspectos psicológicos tem um papel essencial pois estes melhoram a auto-estima, auto-conceito, auto-imagem e diminuição da ansiedade e depressão.

Relativamente à comparação dos três factores (ansiedade, depressão e somatização) e o índice geral de sintomas tendo em conta os diferentes estados civis, o presente estudo apresentou diferenças na depressão de mães divorciadas. Estes resultados corroboram os já obtidos no estudo de Teixeira (1998), onde se constatou que os níveis mais altos de depressão se verificavam nas mulheres divorciadas quando comparadas às mulheres solteiras, em união de facto e casadas.

\section{CONCLUSÃO}

As conclusões específicas deste estudo no que se refere aos objetivos do mesmo são limitadas. No entanto, foi possível, com base no comportamento das médias, constatar que quanto maior for a sobrecarga de trabalho e emocional das mulheres piores serão os seus índices de saúde mental. De particular relevância, e com implicações clínicas, foi a constatação que as mães que praticam atividade física com regularidade apresentam melhores níveis de saúde mental, tal como esta foi avaliada pelas manifestações sintomáticas de somatização, ansiedade, e índice geral de saúde. pois não existem diferenças significativas relativamente aos resultados obtidos com excepção da influência da prática da actividade física. Perante este facto, é importante referir o interesse de realização de futuras investigações devendo-se aprofundar certas variáveis pela tendência dos valores que o 
nosso estudo apresenta.

Ao longo da realização do estudo deparamo-nos com algumas dificuldades, tais como a exigência de mais tempo para a aplicação dos questionários, comparativamente a outras faixas etárias, já que as mães tinham de ter necessariamente um filho adolescente.

Com base nos resultados obtidos os autores recomendam que devem ser realizadas campanhas de sensibilização para uma maior adesão à prática de exercício físico, junto à comunidade em geral.

\section{Agradecimentos:}

Nada declarado.

\section{Conflito de Interesses:}

Nada declarado.

\section{Financiamento:}

Nada declarado.

\section{REFERÊNCIAS}

American Psychiatric Association (2006). DSM-IV-TR. Manual de Diagnóstico e Estatística das Perturbações Mentais. Texto Revisto. Lisboa: Climepsi Editores.

Araújo, S., Mello, M., \& Leite, J., (2007). Anxiety disorders and physical exercise. Revista Brasileira Psiquiátrica, 29(2), 164-171. doi:10.1590/S151644462006005000027

Borsa, J.C., \& Grendene., F. (2008). Conflitos éticos na psicoterapia com adolescentes. Revista Electrónica da Ulbra São Jerónimo, 3, 1-10.

Brito, H.(2006). Estresse, resiliência e vulnerabilidade: comparando famílias com filhos adolescentes na escola. Crescimento Desenvolvimento Humano, 16(2), 25-37.

Caspersen, C.J., Powell, K., \& Christenson, G. (1985). Physical activity, exercise, and physical fitness: Definitions and distinctions for health-related research. Public Health Report, 100(2), 126-132.

Cheik, N., Reis, I., Heredia, R., Ventura, M., Tufik, S., Antunes, H., \& Mello, M., (2003). Efeitos do exercício físico e da atividade física na depressão e ansiedade em indivíduos idosos. Revista Brasileira Cinética e Movimentos, 1 (3), 45-52.

Coates, V. (S.D). Transformações na família no transcorrer da adolescência dos filhos. Adolescência Latinoamericana, pp. 40-46. Manuscrito não publicado.

Coêlho, N.L., \& Tourinho, E.Z. (2008). O Conceito de ansiedade na análise do comportamento. Psicologia: Reflexão e Crítica, 21(2), 171-178. doi: 10.1590/ S0102-79722008000200002

Doron,R., \& Parot, F.(2001). Dicionário de Psicologia. Lisboa: Climepsi Editores.

Feldman, R., Olds, S., \& Papalia, D. (2001). O mundo da criança. Portugal: McGraw-Hill de Portugal.

Vasconcelos-Raposo, J., Fernandes, H., Mano, M., Martins, E., \& (2009). Relação entre exercício físico, depressão e índice de massa corporal. Motricidade, 5(1), 21-32.

Freitas, M.(2009). Ansiedade nas avaliações escolares: Uma abordagem psicoterapêutica sob estados modificados de consciência num grupo de alunos universitários. Universidade da Madeira, Madeira.

Jatobá, J., \& Bastos, O. (2007). Depressão e ansiedade em adolescentes de escolas públicas e privadas. Revista Brasileira Psiquiátrica, 56(3), 171-179. doi: 10.1590/ S0047-20852007000300003

Mendes, A., Loureiro, R., \& Crippa, S. (2008). Depressão materna e a saúde mental de escolares. Revista Psiquiátrica Clínica, 3(5), 178-86.

Prochaska, J., Sallis, J., \& Long, B. (2001). A physical activity screening measure for use with adolescents in primary care. Archives of Pediatric Adolescence Medicine, 155, 554-599. 
Salve, M.(2007). A prática da atividade física: Estudo comparativo entre os alunos de graduação da UNICAMP. Revista de Desporto e Saúde da Fundação Técnica e Científica do Desporto, 4(3), 41-47.

Teixeira, J.M. (1998). A depressão e a mulher na sociedade moderna. Revista Psiquiátrica, $11(3), 51-53$.
Wiesner, M., Chen.,V., Windle, M., Elliott, M.N., Grunbaum, J.A., \& Kanouse, D.E.(2010). Factor structure and psychometric properties of the brief symptom inventory-18 in women: A MACS Approach to testing for invariance across racial/ethnic groups. Psychological Assessment, 22(4), 912- 922. doi: 10.1037/ a0020704. 